\title{
Richard III, la Bretagne et Henry Tudor ( 1483-1485)
}

\section{C.S.L. Davies}

\section{Citer ce document / Cite this document :}

Davies C.S.L. Richard III, la Bretagne et Henry Tudor ( 1483-1485). In: Annales de Bretagne et des pays de I'Ouest. Tome 102, numéro 4, 1995. pp. 33-47;

doi : 10.3406/abpo.1995.3838

http://www.persee.fr/doc/abpo_0399-0826_1995_num_102_4_3838

Document généré le 22/05/2016 


\title{
Résumé
}

Les relations entre l'Angleterre, la Bretagne et la France furent d'une très grande importance sur la période 1483-1485. Leur principale conséquence fut l'appui donné par la France à Henry Tudor, alors en exil, expédition qui lui permit d'envahir l'Angleterre en 1485, au cours de laquelle le roi Richard III fut vaincu et tué, et enfin d'accéder au trône sous le nom d'Henry VII. Un document d'une valeur historique extrême a été mal daté (1484 au lieu de 1485), donnant ainsi l'illusion que Richard III s'était engagé davantage, et plus tôt, dans l'alliance avec la Bretagne, qu'il ne le fut en réalité. À la lumière de cette nouvelle information, l'auteur réinterprète les événements complexes des années 1483-1485, en rendant compte des rapports qui évoluèrent tout au long de la période entre le régime d'Anne de Beaujeu et l'opposition princière en France, entre Landais et ses adversaires en Bretagne, entre l'archiduc Maximilien et les cités flamandes. La raison pour laquelle la France fournit une aide à Henry Tudor était qu'elle redoutait une alliance entre Richard et la Bretagne. Cette alliance, qui ne fut en fait pas réalisée avant 1485, pourrait ne pas avoir été envisagée sérieusement, et fut immédiatement rendue nulle par la chute de Pierre Landais.

\begin{abstract}
Relations between England, Brittany and France in 1483-5 were of crucial importance, leading to French support of the exiled Henry Tudor for his invasion of England in 1485, the defeat and death of Richard III, and Henry 's becoming king as Henry VII. A vital document has been wrongly dated, to 1484 rather than 1485 ; and this has given the impression that Richard III was far more committed to the Breton alliance, and from an earlier date, than was infact the case The author re-interprets the complex events of 1483-5 in this light, involving as they do the ever-changing relationships of the Beaujeu regime and the princely opposition in France, of Landais and his opponents in Brittany, and the archduke Maximilian and the Flemish dues. French support for Tudor was based on fear of an alliance between Richard and Brittany which was not infact achieved until 1485, which may not have been seriously intended, and which was immediately negated by the fall of Landais.
\end{abstract}




\section{Richard III, la Bretagne et Henry Tudor (1483-1485)}

Les relations entre l'Angleterre, la Bretagne et la France furent d'une très grande importance sur la période 1483-1485. Leur principale conséquence fut l'appui donné par la France à Henry Tudor, alors en exil, expédition qui lui permit d'envahir l'Angleterre en 1485, au cours de laquelle le roi Richard III fut vaincu et tué, et enfin d'accéder au trône sous le nom d'Henry VII. Un document d'une valeur historique extrême a été mal daté (1484 au lieu de 1485), donnant ainsi l'illusion que Richard III s'était engagé davantage, et plus tôt, dans l'alliance avec la Bretagne, qu'il ne le fut en réalité. À la lumière de cette nouvelle information, l'auteur réinterprète les événements complexes des années 1483-1485, en rendant compte des rapports qui évoluèrent tout au long de la période entre le régime d'Anne de Beaujeu et l'opposition princière en France, entre Landais et ses adversaires en Bretagne, entre l'archiduc Maximilien et les cités flamandes. La raison pour laquelle la France fournit une aide à Henry Tudor était qu'elle redoutait une alliance entre Richard et la Bretagne. Cette alliance, qui ne fut en fait pas réalisée avant 1485 , pourrait ne pas avoir été envisagée sérieusement, et fut immédiatement rendue nulle par la chute de Pierre Landais.

Relations between England, Brittany and France in 1483-5 were of crucial importance, leading to French support of the exiled Henry Tudor for his invasion of England in 1485, the defeat and death of Richard III, and Henry's becoming king as Henry VII. A vital document has been wrongly dated, to 1484 rather than 1485 ; and this has given the impression that Richard III was far more committed to the Breton alliance, and from an earlier date, than was in fact the case The author re-interprets the complex events of 1483-5 in this light, involving as they do the ever-changing relationships of the Beaujeu régime and the princely opposition in France, of Landais and his opponents in Brittany, and the archduke Maximilian and the Flemish cities. French support for Tudor was based on fear of an alliance between Richard and Brittany which was not in fact achieved until 1485, which may not have been seriously intended, and which was immediately negated by the fall of Landais. 
Pendant le bref règne de Richard III, les rapports de l'Angleterre avec la Bretagne et le royaume de France furent d'une importance capitale'. Quand le roi Édouard IV mourut en avril 1483, son fils aîné, âgé de douze ans, lui succéda et fut proclamé roi sous le nom d'Édouard V. Le frère d'Édouard IV, Richard, duc de Gloucester, fut fait Protecteur du royaume pour la durée de la minorité de son neveu. Le 26 juin 1483. Richard lui-même fut proclamé roi sous le nom de Richard III, sous prétexte que son neveu et les autres enfants d'Édouard IV étaient bâtards. Le roi déposé et son jeune frère furent incarcérés à la Tour de Londres. Ils étaient toujours en vie pendant l'été de 1483, lorsqu'on les vit pour la dernière fois, mais, à la fin de l'année, on croyait en général qu'ils étaient morts. Comme il fallait s'y attendre, on accusa leur oncle d'avoir ordonné leur mise à mort. Deux ans plus tard, le 22 août 1485, Richard III fut vaincu et tué à la bataille de Bosworth par les troupes d'Henry Tudor, qui s'empara du trône et prit le nom d'Henry VII.

Henry Tudor s'était réfugié en Bretagne en 1471 après la défaite des forces lancastriennes par le roi Édouard IV. On le garda à vue en Bretagne, où pour un temps le duc François II le traita en prisonnier. En octobre 1483, François lui fournit une armée pour participer à une révolte en Angleterre ; une guerre navale s'ensuivit. Au mois de juin 1484, une trêve fut conclue entre l'Angleterre et la Bretagne. Craignant qu'un accord secret entre le roi Richard et le ministre breton Pierre Landais n'ait été conclu, Henry Tudor et sa suite s'enfuirent vers le $1^{\text {er }}$ octobre 1484 pour se réfugier à la Cour de France. C'est avec l'appui financier, naval et militaire de la France que, en juillet ou en août 1485, Henry fit voile vers l'Angleterre, où il battit le roi Richard à Bosworth. Les relations entre l'Angleterre et la Bretagne sont donc de la plus haute importance pour l'histoire de l'Angleterre à cette époque. Elles n'en sont pas moins importantes pour l'histoire de la Bretagne et de la France, étant donné que les efforts déployés par Henry VII après 1485 pour garantir l'indépendance de la Bretagne à l'égard de la couronne de France furent entravés, du moins temporairement, par les obligations qu'il avait contractées envers le gouvernement français.

Tous ces événements ont été admirablement décrits par l'éminent spécialiste des relations anglo-bretonnes, Pocquet du Haut-Jussé ; des recherches postérieures ont complété le tableau en y apportant des précisions. Malheureusement, Pocquet et les historiens qui lui ont succédé ont été induits en erreur sur un point capital, ce qui les a conduits à se faire une fausse idée des relations anglo-bretonnes pendant cette période décisive. Si l'en croit une source à première vue irréprochable, Richard III aurait, le 26 juin 1484, donné l'ordre de mettre 1000 archers à la disposition de François II, duc de Bretagne. Deux commissions se rapportant à cette affaire et datées du 26 juin (sans indication d'année) sont transcrites sur le 'patent roll' de la seconde année du règne de Richard III. Étant donné que l'année en question commença le 26 juin 1484 pour se terminer le 25 juin 1485, on a naturellement daté les deux commissions du 26 juin 1484. À mon avis, comme je vais le montrer, elles ne

\footnotetext{
1. Une version de cet article a été publiée dans les Nottingham Medieval Studies, XXXVII, (1993), 110-26. Je remercie Monsieur le Professeur Michael Jones, éditeur des Nottingham Medieval Studies, de m'avoir permis de publier cette version française et Yann Tholoniat pour la traduction. Ma reconnaissance pour leur soutien s'adresse à Michael Jones, au $D^{\prime}$ lan Arthurson, à Monsieur le professeur Ralph Griffiths, aux D' Richard Sharpe et D J. L. Watts et surtout au D Pierre Chaplais. Sauf indication contraire, les ouvrages anglais ont été publiés à Londres, et les ouvrages français à Paris.
} 
furent en fait expédiées qu'un an plus tard, en juin 1485, et on les copia à l'époque sur le 'patent roll' de la mauvaise année, erreur qui n'est pas surprenante. parce que les deux documents se rapportent au premier jour de la nouvelle année et qu'ils furent établis en grande hâte. Si l'on accepte cette nouvelle datation, c'est tout le cours des relations anglo-bretonnes qu'il nous faut réinterpréter ${ }^{2}$.

Un des documents en question nomme John Grey, lord Powys, capitaine des mille archers qui doivent être envovés en Bretagne pour secourir le duc, parent de Richard. L'autre donne à Francis, vicomte de Lovell, à William Catesby et à d'autres pleins pouvoirs de rassembler les archers à Southampton ou dans les environs et de faire savoir au roi qu'ils l'ont fait aussi rapidement que possible ${ }^{3}$.

Est-il vraisemblable que les documents aient été mal datés ? Il est certain que la datation traditionnelle de 1484 soulève d'énormes difficultés. Nous n'avons aucune preuve que l'expédition ait été annulée par la suite. Mais si le corps expéditionnaire fut bien envoyé en Bretagne en juin 1484, comment se fait-il qu'on ne trouve aucune trace de ce qui lui est advenu là-bas? Il y a en réalité du côté breton des indices que la date de 1484 est erronée. Le 24 juin 1485, un parti de nobles bretons, avec l'appui de la France, monta un coup contre Pierre Landais, trésorier et premier ministre de facto. Le coup réussit ; Landais fut arrêté le 30 juin et après un simulacre de procès, il fut exécuté sommairement le 19 juillet. Lorsqu'on l'interrogea le 16 juillet sur son projet d'alliance avec les Anglais et avec l'archiduc Maximilien, Landais expliqua que Richard III avait tout d'abord promis un contingent de 4000 archers, mais que finalement il l'avait réduit à mille. Guillaume Guillemet était passé en Angleterre avec sept navires pour les transporter : Guillemet et, implicitement, les navires étaient toujours en Angleterre. Il semble à tout le moins peu probable qu'une flotte de sept navires bretons ait pu rester patiemment à l'ancre à Southampton pendant plus d'un an sans servir à rien et sans attirer l'attention, comme elle l'aurait fait, si tout cela s'était passé en juin 1484 ; il est plus vraisemblable que la promesse d'archers et la mission de Guillemet eurent lieu en 1485 , juste avant l'interrogatoire de Landais ${ }^{4}$.

En eux-mêmes, nous dira-t-on, ces arguments ne sont guère convaincants, lorsqu'il s'agit de mettre en question un document officiel, mais l'examen des 'patent

2. B.-A. POCQUET DU HAUT-JUSSÉ, François II, duc de Bretagne, et l'Angleterre, (1458-1488) (1929), [abrégé désormais Pocquet], p. 259. Cf. James GaIRDNer, Richard III (2e éd., Cambridge, 1898), p. 172 ; Charles Ross, Richard III (1981), p. 199 ; John GILlingham, The Wars of the Roses (1981) p. 234 ; R.A. GRIFFITHS et R.S. ThOMAS, The Making of the Tudor Dynasty (Gloucester, 1985), p. 111,146 (un des rares ouvrages à assumer la thèse d'une réelle expédition maritime) ; Rosemary HORROX, Richard III ; a Study in Service (Cambridge, 1989), p. 309 ; A.J. POllard, Richard III and the Princes in the Tower (Gloucester, 1992), p. 159-60. Ernest LAVISSE, Histoire de la France (9 vol. en 18 parties, 1903-10), iv, $2^{\mathrm{e}}$ partie, p. 432, semble être le seul à situer les archers en 1485 . Il ne cite aucune source, mais semble avoir travaillé directement à partir de l'interrogatoire de Landais.

3. Les deux commissions sont à Londres, Public Record Office, sur les 'patent rolls' de la deuxième année de Richard III C66/558 et C66/559. Elles sont résumées dans les Calendar of Patent Rolls, 1476-85 (1901) p. 517, 547. Pour une discussion détaillée des 'rolls' et des détails supplémentaires, cf. mon article dans les Nottingham Medieval Studies, Appendix I.

4. L'interrogatoire de Landais a été publié d'après les Archives Nationales, K73, n³1, par R. DE MAULDE, Procédures politiques du règne de Louis XII. (Collection de documents inédits, $1^{\text {re }}$ série. iii, 1885), p. 1085-90. 
rolls' eux-mêmes nous confirme que la date traditionnelle de 1484 est inacceptable. Aucune des deux commissions ne se trouve au début du 'roll', mais vers la fin, à la suite de lettres concernant des événements de 1485. Il est clair qu'on ne les copia qu'en juin 1485 ; la hâte avec.laquelle l'affaire fut conclue nous explique comment il se fait que les commissions furent copiées par erreur sur le 'roll' de la seconde année du règne qui venait juste de se terminer au lieu de l'être, comme elles l'auraient dû, sur le 'roll' de la troisième année.

Le récit fait par Landais, au cours de son interrogatoire, des relations entre la Bretagne, l'archiduc Maximilien (qui s'efforçait d'imposer son autorité aux Pays-Bas au nom-de son fils en bas âge, Philippe, héritier du patrimoine de Bourgogne) et Richard III se comprend mieux, si l'on accepte la nouvelle datation. Ce que nous dit Landais des négociations ne nous permet pas de les placer en totalité entre les mois d'avril et de juin 1484. Lorsqu'on l'interrogea pour la première fois sur les «seellez, alliances et obligations d'entre le Roy d'Angleterre, Haultriche et Bretaigne», il nia que le duc ait contracté une obligation formelle. Il avoua cependant qu'Antoine de Longueil, évêque de Léon, était allé en mission auprès de l'archiduc Maximilien et du roi Richard «pour treter l'unyon d'entre eulx», et que l'archiduc avait écrit au duc François «qu'il y entendoit besongner et marier sa sour au Roy d'Angleterre», mais il refusa d'admettre qu'il en savait plus long'.

Interrogé à nouveau, Landais expliqua qu'Orléans et Dunois étaient opposés à l'idée de faire venir des soldats anglais, mais que «en leur absence et a leur desceu» il avait décidé qu'il serait utile de les amener pour décourager les seigneur bretons à l'intérieur du duché, qu'il suspectait d'être en rapport avec d'autres seigneurs bretons en exil à l'extérieur. Il le fit sur la foi de renseignements reçus «du petit Salazan», d'après qui Richard aurait promis 5000 ou 6000 hommes $^{6}$. Landais avait depuis dépêché Jehan Lesquelen en Angleterre pour tâcher de savoir si le roi tiendrait sa promesse. Puis Richard réduisit le nombre promis à 4000 et plus tard à un millier seulement?. C'est alors que Guillemet fut envoyé en Angleterre avec sept vaisseaux pour transporter les troupes; il y était toujours.

5. Sur Longueil, cf. de La Chesnais-Desbois \& Badier, Dictionnaire de la Noblesse, (3e éd., 19 vol., 1880), xii, p. 295-300. Il n'était pas Breton, mais de vieille souche normande, qui s'était solidement retranchée pendant le XVe siècle au Parlement de Paris, atteignant des postes élevés et faisant de beaux mariages. Quelques membres de cette famille entrèrent dans les ordres, suivant l'exemple du grand-oncle d'Antoine, Richard, évêque de Coutances et cardinal. L'oncle d'Antoine, Pierre, avait établi des rapports en Bourgogne en tant qu'évêque d'Auxerre (1449-73). Antoine lui-même, au cours d'une mission diplomatique ultérieure dans les Pays-Bas, engendra un fils, Christophe de Longueil, qui fit une brillante carrière à Paris et à Rome comme humaniste ; cf. P. G. BIEIENHOZ, Contemporaries of Erasmus, (3 vol., Toronto, 1985-7), ii, p. 342-5 (contient aussi une brève notice sur Pierre, le frère d'Antoine).

6. Sur Salazar, cf. A. GoOdMAN et A. MACKAY, «A Castilian Report on English Affairs, 1486», English Historical Review, lxxxviii, (1973), p. 92-9 ; et E. M. NOKES et Geoffroy WHEELER, «A Spanish Account of the Battle of Bosworth», The Ricardian, vol. ii, $n^{\circ} 36,(1972)$, p. 1-5. Le nombre de gens autorisés à voyager dans sa suite entre l'Angleterre et les Pays-Bas au printemps 1485 suggère qu'il eut une carrière d'agent diplomatique, et non seulement de capitaine mercenaire ; Rosemary HORROX et P.W. HAMMOND éd., British Library Harleian Manuscript 433, (4 vol., Richard III Society, Upminster, 1979-83), [abrégé désormais Harl 433], ii, p. 209, 213-4, 216, $217,228$.

7. Je n'ai pas réussi à poursuivre l'histoire de Lesquelen au-delà de la brève référence dans PocQuET, p. 258, où on le dit allié à la famille de Rohan ; mais comme cette famille était grandement divisée, ce renseignement est de peu d'importance. Le texte suggère qu'il alla en Angleterre en tant qu'agent 
À une autre question sur ce qu'il comptait faire avec les soldats anglais et aussi avec les deux mille Suisses que, sur ses ordres, Jean Feuillet était allé recruter, Landais répondit qu'il espérait les utiliser pour combattre les seigneurs bretons rebelles et les Français qui étaient avec eux, et aussi pour assiéger les forteresses d'Ancenis et de Châteaubriant, qui étaient entre les mains des rebelles ${ }^{8}$. Landais décrivit les complots tramés avec Orléans, Alencon et Dunois, et parla d'un plan d'attaque contre les Bretons rebelles à Angers.

À son dernier interrogatoire, le 10 juillet, alors qu'il avait déjà été condamné à être pendu, Landais nia avoir travaillé contre le roi de France ; il avait travaillé seulement contra la faction de Beaujeu. En particulier, il refusa d'admettre que la mission de l'évêque de Léon en Angleterre ait été préjudiciable en quoi que ce soit au roi ou au royaume de France.

Ce qui ressort clairement de l'interrogatoire est que les négociations avaient traîné en longueur et qu'elles avaient souffert de plusieurs changements d'avis de la part de Richard III, et, avant tout, qu'elles s'étaient poursuivies presque jusqu'à la date de l'interrogatoire ( 16 juillet 1485), et même qu'à ce moment précis la flotte de Guillemet attendait toujours pour transporter l'armée anglaise promise par Richard. En outre, Landais sous-entend que l'offre d'archers devait être placée dans le contexte d'une triple alliance entre le duc de Bretagne, Richard III et l'archiduc Maximilien. Comme nous le verrons, ce n'est que pendant l'été de 1484 que l'idée d'une alliance de ce genre commença à germer, et on ne la prit pas au sérieux avant 1485 . Le témoignage de l'interrogatoire concorde donc avec la date révisée de l'expédition des commissions pour l'armée anglaise, celle du 26 juin 1485.

Sur cette base. il nous est désormais possible de réexaminer l'exposé classique des relations de Richard III avec la Bretagne et avec Maximilien'. Jusqu'en 1483, ce que le duc de Bretagne voulait avant tout était de sauvegarder l'indépendance de son duché face au danger représenté par le roi Louis XI. Il lui fallait donc faire appel à l'appui militaire de l'Angleterre. Bien que l'indépendance bretonne fût évidemment dans l'intérêt national de l'Angleterre. cela ne veut pas nécessairement dire que l'aide militaire anglaise serait automatiquement fournie lorsqu'elle était requise. La présence en Bretagne d'Henry Tudor et de son oncle, Jasper Tudor, comte de Pembroke,

de Landais lui-même, et non du duc, ce qui expliquerait le manque apparent de référence dans les archives anglaises. Guillemet fut impliqué dans des missions diplomatiques auprès d'Henry VII en 1488 et 1490 . Un parent, Jean Guillemet, avait été un des gardiens d'Henry en 1482. Cf. PocQUET, p. 248, 259 ; (Dom) Hyacinthe MORICE, Mémoires pour servir de preuves à l'histoire ecclésiastique et civile de Bretagne, (3 vol., 1742-6), iii, p. 427, 583, 658-60.

8. Je n'ai trouvé aucune référence à la mission de Feuillet. Dr Ian Arthurson m'a signalé la présence d'un document aux Arch. dép. du Nord, Lille. B 3522/124818, un mandat signé de Maximilien, pour l'envoi de poudre au capitaine de «notre [altéré en "noz"] batteaulx de bretangne», daté du 25 juin 1485, qui pourrait s'y rapporter.

9. Maximilien réclama le gouvernement de plusieurs provinces au titre de régent pour son tout jeune fils Philippe. Cette revendication fut reconnue par la plupart des provinces, mais fut vigoureusement contestée par les «trois membres des Flandres», les cités de Gand, de Bruges et d'Ypres ; Philippe était entre les mains de ces dernières. Maximilien était en guerre ouverte ou imminente avec ces cités tout au long de ces années; celles-ci comptaient naturellement sur l'appui des Français contre lui. Pour un résumé, cf. W.P. Blockmans, «La Flandre dans le royaume à la fin du XVe siècle», in Bernard Chevalier et Philippe Contamine éd., La France de la fin du XV siècle, (1985), p. 71-89. 
fournissait au duc une excellente arme de chantage, si les rois anglais de la maison d'York donnaient la moindre indication qu'ils seraient prêts à sacrifier la Bretagne dans l'intérêt de bonnes relations avec la France ; car Henry Tudor était le meilleur candidat pour représenter les revendications au trône d'Angleterre du parti lancastrien défait. Les Tudor étaient arrivés en Bretagne par accident ; après la défaite lancastrienne en 1471, ils étaient partis pour la France. En Bretagne, on les surveilla de près pour les empêcher de jouer un rôle actif contre le régime yorkiste en Angleterre. La menace qu'ils représentaient pour le roi d'Angleterre était qu'on pouvait les livrer à Louis XI ; en tant que cousin germain de Jasper Tudor et partisan de longue date, bien que pragmatique, de la cause lancastrienne, Louis pourrait être prêt le cas écheant à donner aux Tudors un soutien formidable. Lorsque Richard III monta sur le trône, la réaction du gouvernement breton avait été de regarder sa succession comme une excellent occasion d'obtenir une aide substantielle de l'Angleterre contre la France, d'autant plus que en le roi Édouard IV en avait déjà offert une avant sa mort au mois d'avril $1483^{10}$.

En conséquence, le duc François répondit au message de bonne volonté de Richard en lui demandant une force armée de 4000 archers pour six mois aux frais de Richard, et une autre de 2000 ou 3000 archers à ses propres frais, pour la défense du duché contre la menace d'une attaque de la part de la France ; faute de quoi il était donné à entendre que François n'aurait pas d'autre alternative que de se soumettre aux demandes de Louis XI qu'on lui livre Henry Tudor, ce qu'il avait jusque-là refusé de faire ${ }^{11}$. Richard refusa. En même temps, la mort de Louis, le 30 août 1483 , mit fin à la menace d'une intervention militaire française immédiate en Bretagne. Les Bretons profitèrent de l'occasion pour faire volte-face et pour s'opposer à Richard. En octobre, le duc fournit hommes et argent à Henry Tudor pour lui permettre de se joindre à la révolte de 'Buckingham', suscitée par ceux parmi les Anglais qui étaient demeurés fidèles à la mémoire d'Édouard IV et de son fils déposé 12 . Si le duc décida de donner son appui aux rebelles, c'était peut-être parce qu'il en voulait à Richard de lui avoir refusé son aide militaire, peut-être aussi parce qu'il ne lui avait pas pardonné d'avoir éliminé Édouard V. Depuis 1481, la main du jeune Édouard avait été promise à Anne, fille (et héritière présomptive) du duc François. L'élimination d'Édouard détruisit les plans que François avait faits pour l'avenir de sa famille et et aussi de son duché13. Après la mort d'Edouard IV, le cours des événements en Angleterre avait été suivi de près en Bretagne. C'est en Bretagne qu'Édouard Woodville, avec quelques partisans et deux navires, s'était réfugié, lorsque Richard de Gloucester s'était fait proclamer Protecteur du royaume. Après l'avènement de Richard, en juin 1483, une sorte d'accord fut conclu en Angleterre entre la mère d'Henry Tudor, Lady Margaret Beaufort et la reine Elizabeth, mère

10. POCQUET, p. 239, 242-8. Sur ce besoin endémique de troupes étrangères pour renforcer l'armée ducale, cf. Michael Jones, «L'armée bretonne, 1449-1491», in Chevalier et CONTAMine, op. cit., p. 147-65, particulièrement p. 150-1.

11. POCQUET, p. 246-7 ; James GAIRDNER éd., Letters and Papers of the Reigns of Richard III and Henry VII (2 vol., Rolls Ser., 1861-3), i, 22-3 (la mission d'Hutton) et 37-43 (celle de Mainbier).

12. Pocquet, p. 248-53 ; Griffiths et Thomas, Tudor Dynasty, p. 102-5.

13. Pocquer, p. 231-2 ; Charles Ross, Edward IV (1974), p. 285. Le duché allait passer au deuxième fils du couple et ne serait pas absorbé par la couronne anglaise. 
d'Édouard V et sœur de Woodville ${ }^{14}$. Il est presque certain que cette alliance eut sa contrepartie en Bretagne sous forme d'arrangement entre Woodville et Tudor. Le duc François (ou Landais) espérait peut-être que l'aide donnée à Tudor amènerait la restauration d'Édouard $\mathrm{V}$ ou, si cela n'était plus possible, qu'elle lui donnerait le plaisir de se venger et de voir s'installer en Angleterre un régime Tudor, qui serait reconnaissant à la Bretagne ${ }^{15}$. En réalité, le résultat immédiat fut un désastre, car Richard triompha de ses ennemis. Henry décida de ne pas débarquer et s'en retourna en Bretagne en passant par la France. Le jour de Noël 1483, les armées vaincues de Woodville et de Tudor renforcèrent leur alliance : Henry promit d'épouser Elizabeth, fille aînée d'Édouard IV ; en échange, les rebelles jurèrent de soutenir ses prétentions au trône. De toute évidence, ils étaient persuadés qu'Édouard $\mathrm{V}$ et son jeune frère étaient morts. Une violente guerre navale éclata dans la Manche ${ }^{16}$.

La situation se modifia une fois de plus au cours de la première moitie de 1484 , en partie peut-être à cause des pertes subies en mer par les Bretons, et aussi en raison des événements en France. Aux États-Généraux qui se réunirent à Tours entre janvier et mars, des querelles s'élevèrent entre Pierre Landais et la régence d'Anne de Beaujeu, celle-ci agissant pour son frère Charles VIII'17. Landais s'allia au parti d'opposition au gouvernement Beaujeu, parti princier dirigé par les ducs d'Orléans et d'Alençon et par le cousin du duc d'Orléans. le comte de Dunois, éminence grise du partil8. D'actives négociations n'en continuèrent pas moins entre les gouvernements français et breton, et l'on se préparait à résister aux Anglais ; il en était encore ainsi au début d'avril 1484' ${ }^{\prime}$. La mort du rival de Landais, l'ancien chancelier de Bretagne déposé, Guillaume Chauvin, survenue en prison le 5 avril, changea tout. Le 7 avril, un groupe de nobles bretons, sous le commandement du prince d'Orange et du maréchal de Rieux, tenta, mais sans succès, de s'emparer du duc de Bretagne et de le forcer à punir Landais. Ce dernier survécut, les conspirateurs prirent la fuite et, le jour de Pâques, 18 avril, Orléans et Dunois arrivèrent à Nantes pour afficher leur soutien pour Landais 20 . Tout comme l'opposition des princes français s'était alliée au gouvernement breton de Landais, le gouvernement régent français se

14. Ross, Richard $I I I$, p. 195, n`8 ; Michael K. Jones et Malcom G. UNDERwood, The King's Mother; Lady Margaret Beaufort (Cambridge, 1992), p. $61-65$.

15. Alain BoucharT, Grandes Chroniques de Bretagne, édité par M.-L. Auger et G. Jeanneau, 2 vol., Sources d'histoire médiévale, 1986, ii, p. 459-60.

16. PoCquet, p. 253-5; MORICE, Preuves, iii, p. 431-2, des ordres de rassemblement pour résister à l'invasion anglaise, datés du 15 décembre 1483 .

17. POCQUET, p. 255-7.

18. J.S.C. BRIDGE, A History of France from the Death of Louis XI, (5 vol., Oxford, 1921-36), i, 112-3. 19. POCQUET, p. 257 ; la régente, toutefois, ne procura pas plus d'hommes ou d'argent que ne l'avait fait Richard.

20. MORICE, Preuves, iii, p. 433-7 ; Antoine DUPUY, Histoire de la réunion de la Bretagne à la France, ( 2 vol., 1880), ii, p. 24-7. Cf. Michael JONES, «Aristocratie, faction et État dans la Bretagne du XVe siècle» in Philippe Contamine éd., L'Etat et les aristocraties, XII ${ }^{e}-X V I I^{e}$ siècles; France, Angleterre, Ecosse, (1989), p. 129-60. Sur de Rieux, ChenA Ye-DesboIs \& BADIER, Noblesse, xvii, p. 169-71. Jean de Chalons, Prince d'Orange, était bien plus qu'un noble breton, possédant une principauté souveraine, et de vastes terres à la fois en France et en Bourgogne. Il était neveu du Duc François. Cf. Mikael HARSGOR, Recherches sur le personnel du conseil du roi sous Charles VIII et Louis XII, (4 vol., Thèse, univ. de Paris IV, 1972, publ. Lille et Paris, 1980), i, p. 769-836. 
déclara pour les rebelles bretons. Landais se remit à envisager une alliance avec l'Angleterre. Une trêve avec l'Angleterre fut convenue le 8 juin, à compter du $1^{\text {er }}$ juillet 1484 jusqu'au mois d'avril 148521 .

Il va sans dire que Richard III accueillit avec plaisir l'occasion de négocier avec la Bretagne. Il était en guerre ou sur le point de l'être sur tous les fronts, ce qu'il devait en grande partie à l'activité effrénée des corsaires anglais ${ }^{22}$. Le seul effort, d'ailleurs peu convaincant qu'il ait fait à l'époque pour opérer un rapprochement avec la France est d'avoir donné à l'évêque Thomas Langton, qui allait présenter au pape les compliments de Richard, pleins pouvoirs de négocier en cours de route une trêve, dont la durée n'était mème pas signifiée ${ }^{23}$. Tout espoir d'installer en Écosse un régime anglais satellite sous la direction d'Alexander Stuart, duc d'Albany, s'était effondré lorsqu' Albany, s'était enfui en Angleterre, laissant cependant une garnison anglaise dans le château de Dunbar. Bien que les Écossais aient envoyé diverses missions pour faire la paix avec l'Angleterre, ils se préparaient aussi à attaquer Dunbar et ils avaient l'espoir de reprendre Berwick, que Richard lui-même avait regagné pour l'Angleterre en 1482. En avril 1484, l'alliance traditionnelle de l'Écosse avec la France fut réaffirmée. Des navires français attaquèrent des navires anglais sur la Manche et au mois d'avril, la communauté marchande de Londres considérait qu'une guerre sur terre avec la France était probable pour l'été25. Des commissions furent expédiées pour mettre le pays sur pied de guerre en mai ${ }^{26}$. En juin et juillet 1484 , de son état-major de Scarborough, Richard prit en mains la direction personnelle des opérations navales à la fois contre les Écossais et contre les Français ; il fut victorieux contre les Écossais, mais deux de ses capitaines, Sir Thomas Everingham et James Nesfield, furent faits prisonniers par les Français ${ }^{27}$. En juillet, les alliés de Richard, Albany et Douglas, furent battus à Lochmaben au cours d'une incursion en Écosse. Les honneurs étant également partagés, les Écossais envoyèrent en Angleterre une ambassade de haut rang, qui conclut à Nottingham, en septembre, une trêve de trois ans avec Richard ${ }^{28}$. Le 11 août, Richard avait fait publier une proclamation rigoureuse interdisant formellement tout acte de piraterie contre toute

21. POCQUeT, p. 259 ; T. RYMER, Foedera, (20 vol., 1704-35), xii, p. 226-7.

22. Richard semble avoir suivi avec retard le conseil de Lord Dynham en septembre 1483, selon lequel il devait restreindre les activités des pirates anglais et entretenir de bonnes relations avec Maximilien et la Bretagne; GAIRDNER, Letters and Papers, ii, p. 1-3.

23. RYMER, Foedera, xii, p. 221-2.

24. Norman MACDOUGALL, James III ; a political study, (Edimbourg, 1982), chap. 8-10, p. 158-234, particulièrement p. 185-8, 208-10.

25. Alison HANHAM, The Celys and their world, (Cambridge, 1985), p. 292.

26. Calendar of Patent Rolls, 1476-85, p. 397-401.

27. Nicholas Pronay et John Cox ed., The Crowland Chronicle Continuations 1459-86, (Richard III Soc., 1986), p. 172-3 ; Rhoda EDwARDS, The Itinerary of King Richard III 1483-85, (Richard III Soc., 1983) p. 21. Ce doit être l'occasion à laquelle se réfere Adrien de But, observateur précis et bien informé, moine de l'abbaye de Dunes sur la côte flamande. Richard conclut un traité avec la Bretagne pour résister aux Ecossais, puis rechercha une trêve avec la France, mais en fut empêché par la capture au large de Douvres de «quosdam nobiles familiares» par les Français; Kervyn de Lettenhove éd., Chronique d'Adrien de But, in Chroniques de l'histoire de la Belgique, 3 vol., Commission Royale d'Histoire, Bruxelles, 1870-76, i, p. 600.

28. MaCDOUGALL, James $I I I$, p. $211-4$. 
nation, à l'exception, il faut le signaler, de la France 29. Deux jours plus tard, des navires et des troupes partirent pour défendre Calais contre une attaque que l'on pensait imminente ${ }^{30}$. Cependant, le 12 août, le gouvernement français continuait, bien que d'une manière plutôt nonchalante, ses préparatifs pour des pourparlers de paix ${ }^{31}$.

En de telles circonstances, il était permis de penser qu'une alliance entre l'Angleterre et la Bretagne présentait des avantages des deux côtés : en ce qui concerne Landais, c'était le renforcement de l'alliance contre Beaujeu : quant à Richard, elle lui permettait de se concentrer sur les affaires de France et d'Écosse. À en croire le témoignage de Landais, ce serait l'archiduc Maximilien qui aurait agi comme intermédaire pour l'alliance. Au début de 1484, probablement avant le mois de mai, Maximilien renvoya en Bretagne Maître Antoine de Longueil, qualifié de conseiller de François, accompagné d'un délégué de son propre gouvernement. Maximilien expliquait à François qu'il répudiait le traité d'Arras, que Louis XI l'avait injustement forcé à accepter en 1482 ; selon ses dires, il avait dû céder parce que les villes flamandes en révolte l'y avaient obligé. Il ajoutait, presque comme une sorte de post-scriptum, qu'il avait envoyé une délégation en Angleterre et que c'était son intention d'y envoyer sous peu une ambassade, parce que les citoyens de Gand, en révolte contre lui, avaient déjà fait de même ; mais il ne prendrait aucune décision sans consulter la Bretagne au préalable ${ }^{32}$. Les hésitations de Maximilien sont à noter ${ }^{33}$. Peut-être Richard n'était-il pas persona grata parmi les autres princes en 1484, ce qui rendait l'occasion qui s'offrait à lui d'un accommodement avec Landais d'autant plus attrayante.

D'après Landais, l'offre d'archers anglais aurait été transmise au gouvernement breton par le «petit Salazan». Salazar était un capitaine espagnol qui s'était battu pour

29. Calendar of Patent Rolls, 1476-85, p. 493-4.

30. Public Record Office, C81/1531/3.

31. P. Pelicier éd., Lettres de Charles VIII, (5 vol., Soc. pour l'hist. de la France, 1898-1905), v, p. 180-1. Des agents anglais avaient auparavant prévenu Henry Tudor que l'intention de Richard en négociant avec les Français était de gagner du temps ; cf. l'accusation de Collinbourne, GaIRDNER, Richard III, p. 186.

32. [T., D. \& J.] Godefroy et Lenglet du Fresnoy éd., Mémoires de Messire Philippe de Comines, (4 vol., Paris et Londres, 1747), iv, p. 139-46. Bien souvent daté de 1483, (e.g. PocQueT, p. 2645), le document renvoie à la prise d'Amersfoort et à la libération de l'évêque David d'Utrecht, qui eurent lieu en janvier 1484 ; cf. Hermann WIESFLECKER, Kaiser Maximilian I (5 vol., Munich, 1971 86), i, p. 169. Longueil fut présenté au siège épiscopal de St. Pol-de-Léon le 12 mai 1484, et prêta serment à François II le 15 juillet ; cf. (Dom) Charles Taillandier, Histoire ecclésiastique et civile de Bretagne, (2 vol., 1750-6), ii, p. Ixi-ii. Puisque le document le qualifie simplement de «maître», il doit précéder son élévation au trône épiscopal.

33. Bien que Maximilien soit souvent considéré comme l'allié de Richard III, leurs relations semblent avoir êté de méfiance mutuelle. Piers Puissant, secrétaire de Maximilien, était en Angleterre en septembre 1483, en avril 1484, et fut bien récompensé par Richard en août $1484 ; \mathrm{Harl} 433$ ii, p. 27, iii, p. 22-3 ; RYMER, Foedora, xii, p. 232. Le chevalier bohémien Nicholas von Poppelau rendit visite à Richard en avril-mai 1484 avec un sauf-conduit du père de Maximilien, l'empereur Frédérick III ; P.M. KENDALL, Richard III (1955), p. 322 ; RYMER, xii. p. 210-1. Mais il n'y a aucune trace de négociations sérieuses avant août-septembre 1484, époque à laquelle Richard négocia à la fois avec Maximilien et avec ses adversaires flamands ; RYMER, xii, p. 231-2, 248-9. Cf. Rosl PHILPOT, Maximilian I and England, 1477-1509, London Univ Ph. D thesis, 1975, p. 62-9, qui décrit les relations comme «ténues», situation due en partie à ce que chaque prince était préoccupé de ses propres rebelles. 
Maximilien aux Pays-Bas jusqu'en 1483 et à nouveau à partir de 1486. Entre-temps, il était en Angleterre au service de Maximilien ; on le connaît, entre autres, comme témoin oculaire de la bataille de Bosworth. À une certaine date en 1484 (certainement avant la fin de septembre), le gouvernement anglais mit un navire à sa disposition pour son passage en Bretagne ${ }^{34}$. Selon Landais l'offre d'archers fut faite lorsqu'Orléans et Dunois étaient absents de la cour de Bretagne : ils l'avaient quittée en mai 1484 pour y revenir l'un (Orléans) pas avant 1486 et l'autre (Dunois) en septembre 148435. Il semble bien que l'offre initiale d'archers fut faite entre mai et septembre 1484, formant sans doute partie intégrante d'un marché avec la conclusion de la trêve de courte durée au mois de juin. Mais ce n'était encore qu'un leurre destiné à pousser les Bretons à faire d'autres concessions. Aucun archer ne fut fourni, mais la nouvelle de l'offre parvint à la cour de France. Au mois de septembre, le gouvernement régent chargea Dunois lui-même d'enquêter sur des bruits que 6000 archers étaient arrivés ${ }^{36}$.

L'objectif de Richard était de se saisir d'Henry Tudor. Des agents passaient la Manche dans les deux sens avec des cadeaux et des promesses. Longueil, devenu évêque de St-Pol de-Léon, s'est peut-être rendu en Angleterre ${ }^{37}$. Le Grand Écuyer de Richard (probablement James Tyrrel) était à Vannes en septembre 148438. Au mois d'août, un navire envoyé par le gouverneur de Jersey et transportant des nouvelles pour Landais fut capturé par les Français ${ }^{39}$. En septembre, Henry reçut un message de la part de l'évêque John Morton, qui, bien qu'en exil aux Pays-Bas, était bien informé de ce qui se passait dans les milieux gouvernementaux anglais ${ }^{40}$. Aux envi-

34. Sur Salazar, cf. (6) supra.

35. Yvonne LaBANDE-MaIlfert, Charles VIII et son milieu (1470-1498), (1975), p. 55-6.5 ; Bernard Quilliet, Louis XII, Père du Peuple, (1986), p. 89-94, 104-5.

36. A, Bernier éd., Procès-verbaux des séances du conseil de régence du roi Charles VIII, (Collection de Documents Inédits, $1^{\text {re }}$ sér., t. 2, 1836), p. 100.

37. POCQUET, p. 259-60 ; GRIFFTHS et ThOMAS, Tudor Dynasty, p. 111-2 ; ROSS, Richard III, p. 1969. Taillandier, Hist. de Bretagne, ii, p. 151, indique la présence de Longueil en Angleterre en septembre 1484 pour alerter Morton, mais aucune des sources qu'il cite, que ce soit Polydore Vergil ou D'Argentré, ne mentionne Longueil spécifiquement ; cf. VERGIL, Three Books, éd. par H. ElLIS, Camden Ser, xxix, 1844, p. 205-6, et Bertrand D'Argentre, L'Histoire de Bretaigne, ( $2^{\mathrm{e}}$ éd., Paris, 1605), p. 1057. À partir de Taillandier il trouve une piste dans J. Allanic, Le Prisonier de la Tour d'Elven, (Vannes, 1909), p. 51. De très nombreuses sources contenues dans des chroniques bretonnes tournent à la confusion en situant la rébellion «Buckingham» en octobre 1484, et doivent donc comprimer les événements entre celle-ci et Bosworth en moins d'une année. La question de savoir si Landais était réellement en train de négocier en Angleterre en septembre devra rester ouverte.

38. On affirme parfois que William Catesby fut l'agent de Richard en Bretagne ; cf. GRIFFITHS et Thomas, Tudor Dynasty, p. 111 , et Horrox, Richard III, p. 278. Aussi, Henry Marsille, Vannes au Moyen-Age ( = Bulletin de la Soc. Polymathique de Morbihan, 109, Vannes, 1982), p. 114.

39. PoCQueT, p. 260 ; Bibl. Nat. MS fr. 15538 f.133, cité par Alfred SPONT, «La marine française sous Charles VIII», Revue des Questions Historiques, 55, (1894), p. 387-454, à la p. 392.

40. Griffiths et Thomas, Tudor Dynasty, p. 112-18 ; C.S.L. Davies, «Bishop John Morton, The Holy See, and the Accession of Henry VII», English Historical Review, cii, (1987), 1-30, aux p. 6-7. Le témoignage de Margaret Condon, selon lequel le neveu de Morton, Robert Morton, était avec Henry en Bretagne est un autre indice de valeur; «the Kaleidoscope of Treason», The Ricardian, vii, $n^{\circ} 92$, (1986), p. 208-12. Sur la présence de Tudor en France, cf. A.V. ANTONOVICs, Henry VII, King of England, «By the Grace of Charles VIII of France», in Kings and Nobles in the Later Middle Ages; a Tribute to Charles Ross, édité par R.A. Griffiths et James ShERBONE (Gloucester and New York, 1986), p. 169-84. 
rons du ler octobre 1484, Henry s'enfuit en France, échappant de près au sort que Landais lui avait réservé. Quelques jours plus tard, tous les autres exilés anglais furent autorisés à le suivre (grâce à l'intervention du duc François, qui, parait-il, contremanda les ordres de Landais) ${ }^{41}$.

Avec la fuite d'Henry, la raison immédiate pour une aide militaire ou financière à la Bretagne disparaissait. Richard pouvait se permettre d'oublier ses promesses temporairement. Henry avait peu de chance d'obtenir l'assistance militaire de la France dans un avenir prochain. La cour de France était trop occupée par la lutte pour le pouvoir entre Orléans et Anne de Beaujeu. S'il y avait des forces à épargner, les cités flamandes, qui étaient maintenant en guerre ouverte avec Maximilien, avaient la priorité : après elles venaient les rebelles bretons, qui avaient fait appel à la couronne de France contre le gouvernement ducal de Landais et avaient promis de reconnaître Charles VIII comme le successeur légitime de François aux dépens des filles de ce dernier ${ }^{42}$. Ce n'est qu'en novembre 1484, avec la défection de la garnison de Hammes dans le 'Pale' de Calais, à la cause d'Henry (ce qui indiquait qu'il était bien son propre maître, plutot qu'un simple pantin de Woodville) que l'on commença à envisager une invasion de l'Angleterre par Tudor avec l'appui de la France comme une possibilité sérieuse ${ }^{43}$. La proclamation de Richard contre Henry fut publiée le 7 décembre, des mesures de défense furent mises en train et des efforts faits pour gagner les alliés d'Henry, sans oublier l'ancienne reine. Elizabeth Woodville, et ses enfants ${ }^{44}$.

L'évêque Longueil fut renvoyé en Angleterre pour négocier la prolongation de la trêve anglo-bretonne (et sans doute pour demander à Richard la mise à exécution de sa promesse d'aide militaire) le 6 décembre 148445 . Un peu plus tard, probablement à la fin de janvier 1485, Maximilien proposa une triple alliance de grande envergure entre la Bourgogne, la Bretagne et l'Angleterre ${ }^{46}$. C'est peut-étre en cette occasion, mentionnée par Landais, que Maximilien offrit à Richard sa sœur Kunigunde en mariage ${ }^{47}$. Malgré cela, Richard n'était pas pressé de se décider. De

41. Polydore VERGIL, tout à fait la version 'officielle' de Tudor, ne fait pas mention d'une force militaire anglaise envoyée par Richard III ; Three Books, p. 205-6.

42. BRIDGE, France, i, p. 113-22; WIESFLECKER, Maximilian, i, p. 171-4.

43. Davies, «Morton», p. 7.

44. James Gairdner ed., The Paston Letters (6 vol., 1904), vi, p. $81-4$; Davies, «Morton», p. 9-11 ; HORROX, Richard III, p. 293-8.

45. POCQUET, p. 266.

46. Gairdner, Letters and Papers, ii, p. 3-51. Ce document doit être postérieur à la prise de Dendermonde et de Oudenaarde par Maximilien, respectivement le 26 novembre 1484 et le 4 janvier 1485 ; voir p. 18 et cf. Kervyn de LeTtENhOve, Histoire de Flandre (6 vol., Bruxelles, 1847 50), v, p. 360-3, et WIESFLECKER, Maximilian, i, p. 172. Il est surprenant que, bien que Maximilien ait appris la fuite d'Henry Tudor en France (p. 48), il parle de fugitifs anglais en Bretagne (p. 4). Sans doute le document est-il un brouillon, modifié par la suite et demeuré sans effet. Cf. PHILPOT, «Maximilian I, 1477-1509», p. 67.

47. Sur la sœur unique de Maximilien, Kunigunde, qui sera bientôt mariée au Duc Albert IV de Bavière, cf. WIESFLECKER, Maximilian, i, p. 72, 143, 251-2. Plusieurs mariages avaient été envisagés pour elle, parmi lesquels un avec le futur Charles VIII, et un autre avec le sultan Bayazid, a condition qu'il se convertît au catholicisme. Il semble que l'epouse de Richard, la reine Anne, soit tombée malade peu après l'Epiphanie, le 6 janvier 1485 ; Pronay et Cox, Crowland, p. 174-5. Dupuy, Réunion, ii, à la p. 33, pense que Maximilien avait l'intention d'épouser la sœur de Richard, la duchesse Marguerite de Bourgogne, belle-mère de sa première épouse décédée, la duchesse Marie. Mais cela semble être une lecture impossible du témoignage de Landais. 
toute façon, une offensive militaire était impraticable, étant donné les graves problèmes financiers auxquels il avait à faire face ${ }^{48}$. Pour le moment, on pouvait espérer que le gouvernement français de régence serait renversé après l'appel du duc d'Orléans au Parlement de Paris en janvier 1485, ou au moins que la guerre civile pourrait éclater en France avec des troupes bretonnes engagées au service d'Orléans ${ }^{49}$. Dans ces circonstances, il n'y aurait pas besoin d'une intervention anglaise. Cependant en février, Charles VIII et la régente firent leur entrée triomphale à Paris ; mais ce n'est que le 23 mars, lorsqu'une nouvelle réconciliation provoqua le retour d'Orléans au Conseil, que la situation en France devint suffisamment stable pour qu' on puisse envisager une fois de plus une invasion de l'Angleterre par Tudor avec l'appui de la France ${ }^{50}$. Le 4 mai, la couronne de France se décida finalement à épouser la cause d'Henry ${ }^{51}$.

La longue trêve entre l' Angleterre et la Bretagne, qui devait durer jusqu'en 1492, fut conclue le 7 mars. Étant retourné en Bretagne, où François ratifia la trève le 9 avril, Longueil repartit pour l'Angleterre le 11 avril. C'est vraisemblablement à cette époque que le gouvernement anglais prit l'engagement définitif de fournir une aide militaire. Guillemet et sa flotte furent dépêchés en Angleterre pour ramener les archers anglais promis, quelque temps avant le renversement de Landais à la fin de juin, et probablement avant l'expédition réelle des commissions pour la revue des archers, le 26 juin $1485^{52}$. L'alliance militaire discutée en 1484 était enfin effectuée.

Ce scénario soulève un autre problème. Comment Richard pouvait-il mettre de côté mille hommes pour la Bretagne le 26 juin 1485, à une époque où ses ressources étaient déployées pour faire face à l'invasion attendue de Tudor ? Le 22 juin. Richard avait ordonné une mobilisation générale contre l'invasion : le lendemain, il publiait à nouveau, avec un supplément, la proclamation contre Henry et ses adhérents qui avait été lancée la première fois en décembre 148453. George Neville était en mer avec un contingent de soldats ( $y$ compris des archers) depuis le mois d'avril, Lovell était occupé à renforcer les défenses de la côte sud et, semble-t-il, à coordonner les mesures défensives ${ }^{54}$. Ce ne semblait guère être le moment d'envoyer des troupes à l'étranger, quand on se rappelle la répugnance de Richard à le faire à l'époque antérieure. Et une fois la décision prise, pourquoi tant de hâte à l'exécuter ?

48. Horrox, Richard III, p. 305-10. Ceci n'empêcha pas l'agent de Maximilien de proclamer à Calais le 10 avril 1485 que la guerre avait été déclarée entre l'Angleterre et la France, et que Richard envoyait à Maximilien 10000 archers ; de BUT, Chronique, i, p. 625-6.

49. BRIDGE, France, i, p. 122-6.

50. Richard avait toujours suffisamment d'espoir d'arriver à des relations pacifiques avec la France pour envoyer à Caen chercher des pierres pour ses bâtiments le 12 avril 1485 ; Harl 433, ii, p. 214 ; et pour garantir un sauf-conduit à trois marchands normands à la requête de l'amiral français le 18 avril ; RYMER, Foedera, xii, p. 267.

51. Bridge, France, i, p. $126-7$; P. Pelicier, Essai sur le gouvernement de la dame de Beaujeu, (Chartres, 1882 ; rééd. Marseille, 1983), p. 98-9.

52. Pocquet, p. 267. De toute évidence, Longueil alla aux Pays-Bas ; il était présent à la soumission officielle de Gand à Maximilien le 22 juillet ; cf. G. Doutrepont et O. Jodogne éd., Chroniques de Jean Molinet, (3 vol., Bruxelles, 1935-37), i, p. 467.

53. Harl 433, ii, p. 229-30 ; GAIRDNER, Paston Letters, vi, p. 81-4.

54. Calendar of Patent Rolls, 1476-85, p. 545 ; Pronay et Cox, Crowland, p. 176-7. 
Ici encore, c'est la situation à l'extérieur de l'Angleterre qui nous fournit l'explication. À l'époque, le réseau de Maximilien se resserrait autour des villes flamandes. Bruges et Gand tombèrent toutes les deux entre ses mains en juin 1485. L'armée française envoyée pour secourir Gand dut se replier, plutôt ignominieusement, le 11 juin ${ }^{55}$. Du côté breton, le 21 avril, le gouvernement ducal avait repris sur les rebelles le château d'Ancenis (opération pour laquelle Landais avait à l'origine espéré utiliser les archers anglais) ${ }^{56}$. Mais les rebelles bretons continuaient à tenir à Angers, juste de l'autre côté de la frontière avec la France, et pouvaient passer à l'attaque d'un moment à l'autre. Je suis porté à croire que Richard a pu bluffer et que les archers faisaient partie de ses forces défensives (il est significatif que ce soit à Lovell que la revue des troupes ait été confiée), mais qu'il fallait qu'il fasse semblant de donner à Landais un soutien effectif. Ou bien Richard a pu penser que cela vaudrait la peine d'envoyer mille archers en Bretagne pour empêcher le gouvernement breton de conclure un marché avec les Français, et peut-être même pour encourager les Bretons à attaquer Angers, détournant ainsi les Français de toute entreprise immédiate contre l'Angleterre ${ }^{57}$. Après tout, l'armée française qui s'était repliée de Gand pourrait être utilisée pour attaquer Calais (on savait que c'était l'ambition de son commandant, Philippe de Crèvecœur, seigneur d'Esquerdes, de reconquérir Calais) ${ }^{58}$. Ou bien on pourrait s'en servir, comme il semble bien qu'on l'ait fait en partie, pour renforcer Henry Tudor. L'envoi d'un millier d'archers ou au moins la promesse de leur envoi serait un prix qu'il valait la peine de payer pour empêcher ces éventualités en créant des difficultés à l'intérieur pour le gouvernement français 59 .

Ce qu'il advint exactement des archers, à supposer qu'ils aient jamais été rassemblés, n'est pas connu ; nous ne savons pas grand chose non plus du sort de la flotte de Richard pendant et après l'invasion d'Henry60. Ce qu'on sait mieux, c'est la façon dont Henry réussit à éviter la flotte, à débarquer dans le comté de Pembroke et à marcher sur Bosworth. Après la bataille de Bosworth, il semble bien qu'il n'ait rencontré aucune résistance armée dans les comtés du sud. Les troupes de Richard, s'il y en avait dans la région, s'étaient pour ainsi dire évaporées ; peut-être la mort

55. De Lettenhove, Flandre, v, p. 372-3 ; Blockmans, «La Flandre», p. 82, estime l'armée française à 500 lanciers et à 4000-7000 hommes d'infanterie.

56. DupuY, Réunion, ii, p. 63 ; BRIDGE, France, i, p. 157-8.

57. DupuY, Réunion, ii, p. 65-8. Sur la mission mystérieuse à Jersey en mai 1485 , sans doute pour quelque mission de reconnaissance en Bretagne, de Thomas Hutton, agent de confiance du Roi versé dans les affaires bretonnes, cf. C.S.L. DAVIES, «Richard III, Henry VII and the Island of Jersey», The Ricardian, ix, $\mathrm{n}^{\circ} 119,1992$, p. 334-42.

58. Sur d'Esquerdes, cf. Harsgor, Conseil, ii, p. 1077-1116;p. 1094 sur ses desseins sur Calais. En janvier-février 1485, alors qu'il levait son armée en Artois, DE But rapporte que personne ne savait si c'était en vue d'aider l'armée d'Henry à reprendre Hammes, d'aider Henry lui-même contre Richard, ou de soutenir les cités flamandes contre Maximilien ; Chroniques, i, p. 602.

59. Les historiens anglais ne tiennent pas suffisamment compte de l'inquiétude du gouvernement français en lutte avec les princes et leurs alliés bourguignons (i.e. Maximilien) et bretons. Le soutien à Tudor et la déstabilisation du règne de Richard ne furent qu'un des stratagèmes qu'ils utilisèrent, mais son importance se révéla par la suite.

60. Colin F. RiChMOND, «English Naval Power in the Fifteenth Century», History, lii, (1967), p. 1 15 ; C. F. Richmond, Royal Administration and the Keeping of the Seas, 1422-1485, Oxford D. Phil thesis, 1963, p. 490-536. 
du roi et l'impopularité de ses adhérents dans le sud ne leur offraient-elles guère d'autre solution.

Une révolution tout aussi inattendue se produisit de l'autre côté de la Manche. Le 24 juin vit les craintes de Landais se réaliser. Partant de France, les Bretons dissidents envahirent le duché ; l'armée qu'on avait levée pour les repousser refusa de se battre et exigea qu'on livre Landais à sa merci. Son procès s'ensuivit. Le 16 juillet, c'était l'interrogatoire et le 19 sa pendaison du haut des remparts du château de Nantes. Le 9 août, un traité de paix fut conclu entre la France et la Bretagne61. C'était la victoire des armes de la France et de ses intrigues sur deux fronts, en Bretagne d'abord et peu après, avec la bataille de Bosworth, en Angleterre.

L'armée fantôme d'archers anglais, si c'est bien ainsi qu'il convient de l'appeler, laissa tout de même un écho de son existence. Depuis les États Généraux de janvier 1484, les Français avaient craint une attaque de Richard III. Ces craintes, fondées selon toute probabilité sur son opposition connue à la paix de 1475 entre la France et l'Angleterre, se voyaient renforcées par le projet de corps expéditionnai$\mathrm{re}^{62}$. Les barons bretons hostiles à Landais dénoncèrent le duc et le trésorier au Parlement de Paris le 25 juin 1485 ; ils «font amas de gens estrangers, tant Anglois que autres, pour mener guerre aux dits Barons» 63 . Le même jour, Charles VIII expédia une lettre annoncant que Richard avait assemblé une grande armée pour envahir la France, que le duc de Bretagne avait aussi mobilisé et que d'autres seigneurs et princes voisins avaient des visées sur le royaume ${ }^{64}$. En 1488, l'amiral de France pouvait se vanter que les marins normands qui formaient l'équipage de la flotte d'henry avaient sauvé la France d'une invasion massive par «plus de $x x^{m}$ angloys en vostre royaume, car le navire [sic] estoit tout prest et advitaille pour passer par de ça les ditz Angloys alors qu'ilz [ $c^{\prime}$ est-à-dire les marins normands] descendent le comte de Richemont au dit pays d'Angleterre». Vraisemblablement, l'amiral faisait allusion à la flotte de Neville, y compris les vaisseaux de Guillemet ${ }^{65}$.

Il semble donc bien que l'aide militaire à la Bretagne projetée par Richard devrait être datée de juin 1485, et non de 1484, et que les documents relatifs à cette affaire ne se trouvent sur le 'patent roll' de la seconde année du règne sous la date présumée du 26 juin 1484 qu'à la suite d'une erreur d'enregistrement tout à fait compréhensible. La nouvelle datation des commissions nous force à réinterpréter l'ensemble des relations entre l'Angleterre et la Bretagne. En 1484, Richard n'était en aucune façon totalement gagné à la cause bretonne. Il y eut cette année-là des négociations au cours desquelles Richard était prêt à offrir une aide militaire à la Bretagne, sans

61. BRIDGE, France, i, p. 128-32.

62. Charles VIII accusa Maximilien de tenter de révoquer le traité d'Arras «à l'aide des Anglois, nos anciens ennemis»; de LETTENHOVE, Flandre, v, p. 365. Il justifia aussi son soutien à Tudor en 1485 en allégant que «les Angloys et autres estrangiers se sont puis nostre nouvel et joyeulx advènement à la Couronne, efforcez faire plusieurs enterprinses»; PELICIER, Gouvernement, p. 254.

63. Morice, Preuves, iii, p. 448-9. Il est à remarquer que l'accusation est au présent, ce qui montre que les soldats n'arrivèrent pas plus tôt.

64. Pelicier, Lettres, v, p. 194-5 ; Pelicier, Gouvernement, p. 256-7.

65. Imprimé par le Comte de H. de LAFFERIERE-PERCY in «La Normandie à l'étranger», à partir d'une collection à Saint-Petersbourg, in Bull. de la soc. des Antiquaires de Normandie, vii, (18745), p. 210-33. Je remercie $D^{r} S$. J. Gunn pour cette référence. 
avoir l'intention de mettre cette offre à exécution avant de savoir s'il en tirerait profit, c'est-à-dire probablement la solution du problème que lui posait Henry Tudor. Il est douteux qu'il ait envisagé l'invasion massive redoutée par les Français. Alors que sa politique étrangère jusqu'au milieu de l'été de 1484 semble agressive, pour ne pas dire imprudente, par la suite on peut la qualifier d'extrêmement prudente : ce sont des allusions, des promesses, mais aucune action tangible. La fuite d'Henry hors de la Bretagne en octobre 1484 fut le résultat de rumeurs et d'indiscrétions sur le cours des négociations ; elle n'avait rien à voir avec la soi-disant arrivée d'archers anglais en Bretagne. Lorsque l'alliance entre Maximilien, Richard III et le trésorier Landais fut finalement consolidée en juin 1485, il était trop tard. Dans les deux mois qui suivirent, deux des trois principaux acteurs connurent une mort violente. Seul Maximilien. qui pour le moment avait triomphé aux Pays-Bas. pouvait se consoler des victoires françaises en Angleterre et en Bretagne. 